\title{
Effect of myocardial infarction on components of fibrinolytic system
}

\author{
E. GIDRON, R. MARGALIT, A. OLIVEN, AND Y. SHALITIN \\ From the Clinical Research Laboratory, Department of Medicine B, Rothschild University Hospital and \\ the Department of Biology, Technion-Israel Institute of Technology, Haifa, Israel
}

A systematic investigation of the fibrinolytic system during the period after acute myocardial infarction in 42 patients showed a progressive reduction of fibrinolytic activity, with the longest lysis times on days 4 to 5 after the event. There is a concomitant rise in the levels of fibrinogen, plasminogen, and antiplasmin; only the latter parallels the delay in lysis. After 12 to 15 months, normal lysis times and fibrinogen levels were found in 24 patients re-examined. It is concluded that the reduced fibrinolytic activity in these patients was a transient phenomenon after myocardial infarction.

During the past two decades several investigators have sought to determine the role, if any, of defective fibrinolysis in the pathogenesis of myocardial infarction and coronary artery disease. While some of them (Hume, 1958; Ogston, 1962; Chakrabarti et al., 1966, 1968; Bick et al., 1972; Svehla, 1973; Bruhn et al., 1974) found that there was reduced fibrinolytic activity in the blood of patients with recent or old myocardial infarction, and attributed to it a significant role in the course of coronary artery disease, others (Merskey et al., 1960; Naimi et al., 1963; Katz et al., 1963) found no significantly different fibrinolysis in these patients when compared with normal controls. The majority of papers report the results of random samples, without regard to the time after infarction, and without considering the various components of the fibrinolytic system separately. The purpose of the present investigation was to determine systematically the level of fibrinolytic activity of plasma during the period after an acute myocardial infarct, and to analyse the behaviour of several components of the fibrinolytic system during this period.

\section{Patients and methods}

Fibrinolytic activity of plasma was determined in 42 patients with acute myocardial infarction, aged 42 to 75 years. The diagnosis was established by strict clinical, electrocardiographic, and enzymatic criteria. The fibrinolytic activity was determined by two methods on days, $1,2,4,5,6,8,10,14$, and 17 after the acute episode; the blood was drawn, with

Received for publication 14 June 1976 little or no venous compression, between 8 and 9 a.m. from the fasting patients. In 29 of these patients the level of fibrinogen was determined at the same time; in 10 patients the plasminogen level and in 5 the level of antiplasmin.

In 24 of these patients the fibrinolytic activity was determined again 12 to 15 months after the acute infarction.

\section{Methods}

(1) Dilute blood clot lysis time (DBCLT) (Fearnley et al., 1957). The end-point was determined photographically; the normal range in 35 agematched controls was, in our laboratory, $3.9 \pm 1.05$ hours.

(2) Streptokinase-activated lysis time (SALT) (Gidron $e t$ al., to be published). $1 \mathrm{ml}$ citrated blood is centrifuged and $0.1 \mathrm{ml}$ plasma is diluted with $0.8 \mathrm{ml}$ phosphate buffer $(0.15$ molar, $p \mathrm{H}$ 7.4). $3 \mathrm{U}$ of streptokinase (Varidase, Lederle) is added to the diluted plasma, resulting in a concentration corresponding to $30 \mathrm{U} / \mathrm{ml}$ plasma, followed by $10 \mathrm{U}$ thrombin (Upjohn). The resulting clot lyses in normal subjects in $6.9 \pm 1.4 \mathrm{~min}$. This method measures all the factors involved in fibrinolysis except for the activators which are added in excess.

(3) Fibrinogen was determined by the method of Godal (1961).

(4) Plasminogen was determined by the caseinolytic method of Johnson et al. (1969).

(5) Antiplasmin. The known antitrypsin activity of this inhibitor (Schwick et al., 1966) was 
measured.

The enzymatic activity of trypsin on BAEE (benzoyl arginine ethyl ester) as substrate was determined by the method of Schwert and Takenaka (1955) in a Gilford 2400 spectrophotometer at $253 \mathrm{~m} \mu$. 10 to $30 \mu \mathrm{l}$ serum was added to a system composed of trypsin in a concentration of $2 \times 10^{-6} \mathrm{~mol}$, phosphate buffer $\left(0.05\right.$ molar, $p \mathrm{H}$ 8) and $10^{-3}$. mol BAEE. There is an inverse correlation between the proportional activity of trypsin and the antitrypsin level in the serum.

(6) Significance levels were determined by the normal distribution test, except for plasminogen where student's t test was used.

\section{Results}

\section{FIBRINOLYTIC ACTIVITY}

There was a consistent and significant temporary reduction of fibrinolytic activity in all the patients with acute myocardial infarction during the first week.

The lysis time increased progressively, as measured by both methods, until the 4th or 5th day after the infarction, with a gradual return to initial levels or below during the following days. In 35 patients $(83.3 \%)$ the maximal reduction of fibrinolytic activity was found by both methods on the same day; in 7 patients $(16.7 \%)$ there was a divergence by 1 to 2 days.

(a) Dilute blood clot lysis time (Fig. 1)

The prolongation of the lysis time was significant at its peak $(P<0.05)$ as compared with the values on day 1 after infarction, as well as those of normal controls. The lysis time determined by this method after day 13 was significantly lower than on day 1 .

(b) Streptokinase activated lysis time (Fig. 2)

By this method the lysis time was significantly prolonged in comparison with normal controls from

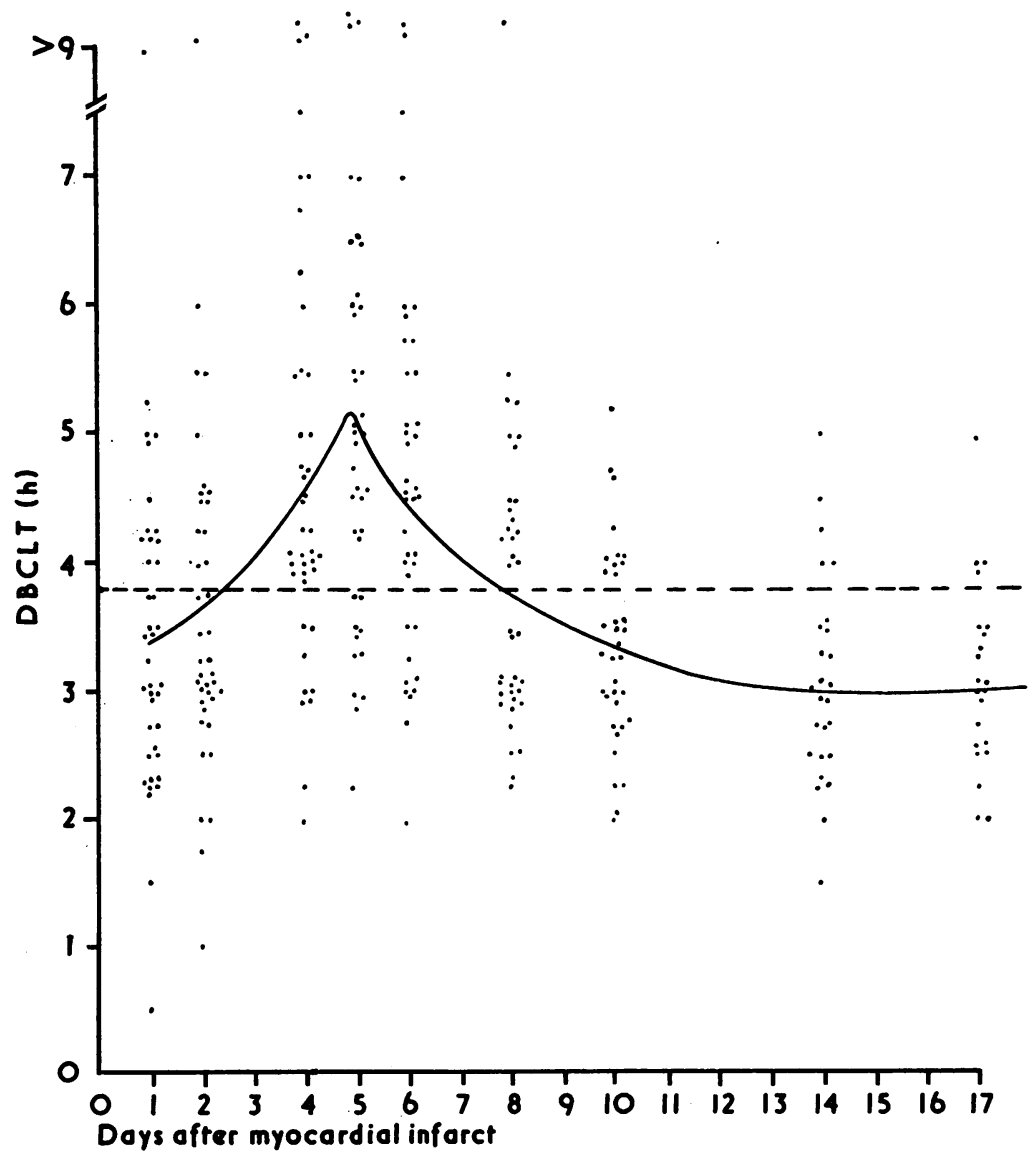

Fig. 1 Dilute blood clot lysis time in 42 patients during the postinfarction period. The broken line represents the mean normal DBCLT $(3.9 \pm 1.05 \mathrm{~h})$ and the continuous line the mean of the observed values each day. 


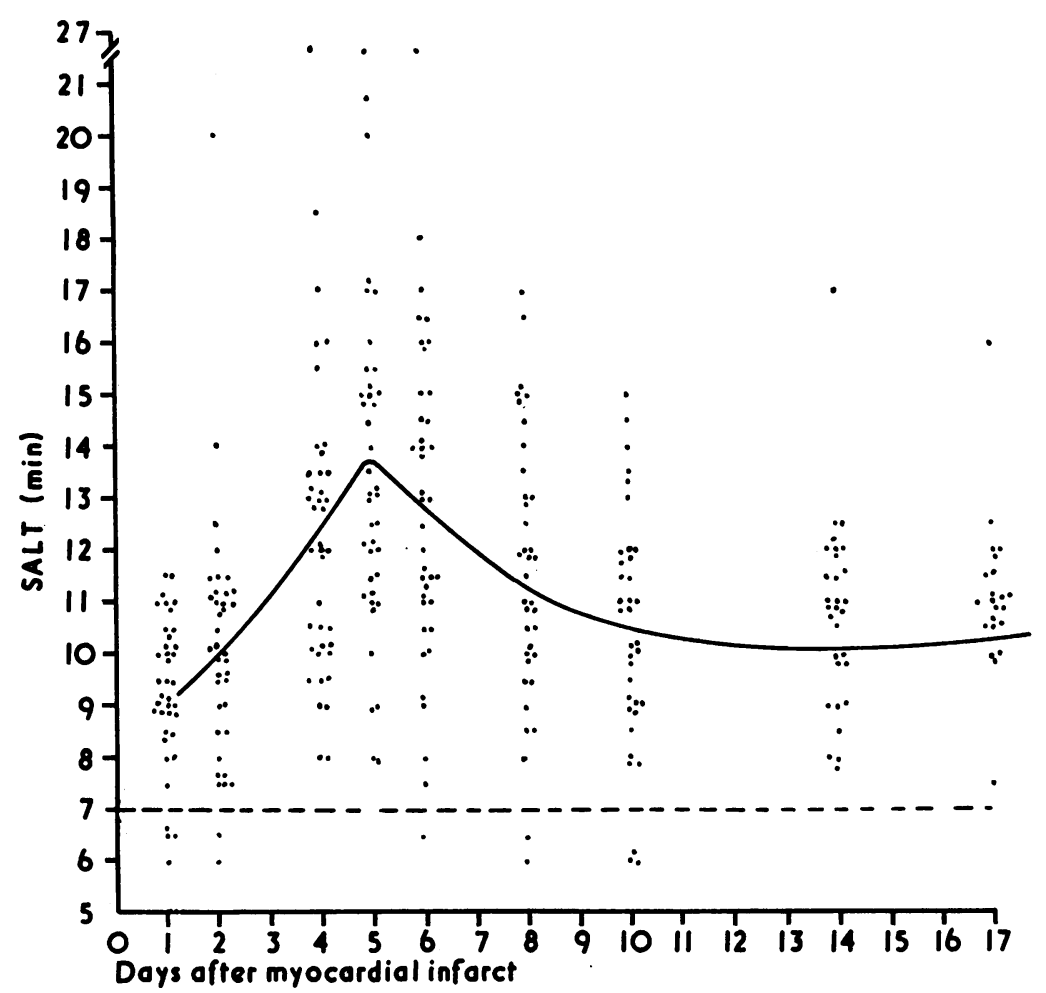

Fig. 2 Streptokinase activated lysis time in 42 patients during the post infarction period. Broken line represents the mean normal SALT (6.9 $11.3 \mathrm{~min})$ and the continuous line the mean of the observed values each day.

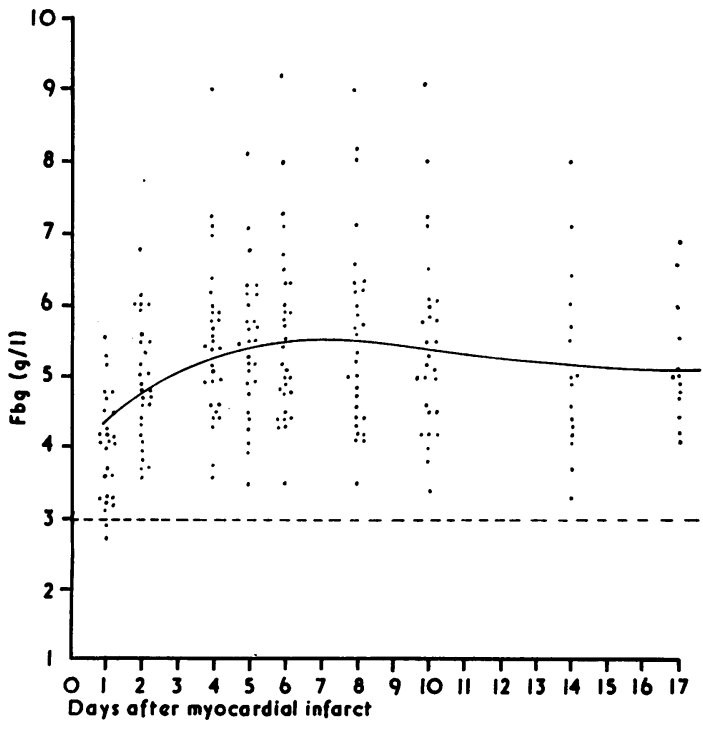

Fig. 3 Changes in plasma fibrinogen (Fbg) level during the post-infarction period. The broken line shows the mean normal level $(3 \pm 1 \mathrm{~g} / \mathrm{l})$ and the continuous line the mean of the observed values each day. day 1 till the end of the acute phase $(P<0.05)$. The maximum prolongation on day 4 or 5 was highly significant both in relation to normal controls and to day $1(P<0.001)$.

\section{FIBRINOGEN (FIG. 3)}

The level of fibrinogen was significantly higher on day 1 after infarction as compared with normal values $(P<0.001)$ and reached its highest values on day $6(P<0.001)$ with a mean rise of more than $2 \mathrm{~g} / \mathrm{l}$ above normal control levels. The fibrinogen level started to decline after day 10 .

\section{PLASMINOGEN (FIG. 4)}

The level of plasminogen rose gradually and the increase was significant from day 5 onwards and reaching a flat peak from days 8 to $10(P<0.05)$. After that there was a gradual decline.

\section{ANTIPLASMIN (FIG. 5)}

The level of these inhibitors rose in the 5 cases in which it was determined on the days after infarction and reached its peak on day 6 , paralleling the re- 


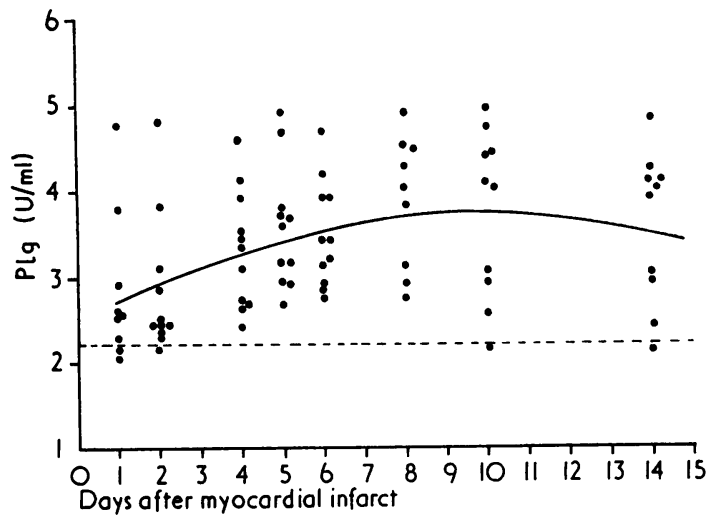

Fig. 4 The plasma plasminogen (Plg) level during 14 days after infarction. The broken line represents the mean normal level $(2.5 \pm 0.9 \mathrm{CTA}$ units $/ \mathrm{ml})$ and the continuous line the mean of the observed values each day.

duction in fibrinolytic activity. The level fell gradually thereafter.

5. Fig. 6 illustrates the changes in the various components of the fibrinolytic system in a single typical patient after myocardial infarction. Whereas the clot lysis time, as measured by the two methods, parallels the rise and fall of the level of inhibitors, there is no direct correlation with the rise in

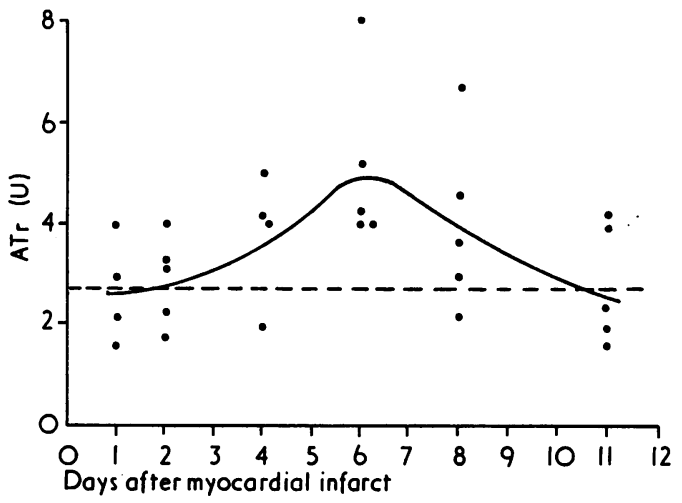

Fig. 5 Antitrypsin level in plasma during 10 days after myocardial infarction. Broken line represents the mean normal level. $A \operatorname{Tr}(U)=$ antitrypsin (arbitrary units).

fibrinogen and plasminogen levels, both the latter being more prolonged.

6. In 2 patients excess fibrinogen and plasminogen were added to the plasma before activation with streptokinase. Fig. 7 shows that nevertheless a delay in lysis time by the SALT method occurs on day 5 after infarction.

7. Twenty-four patients were re-examined 12 to 15 months after the infarction, and the fibrinolytic

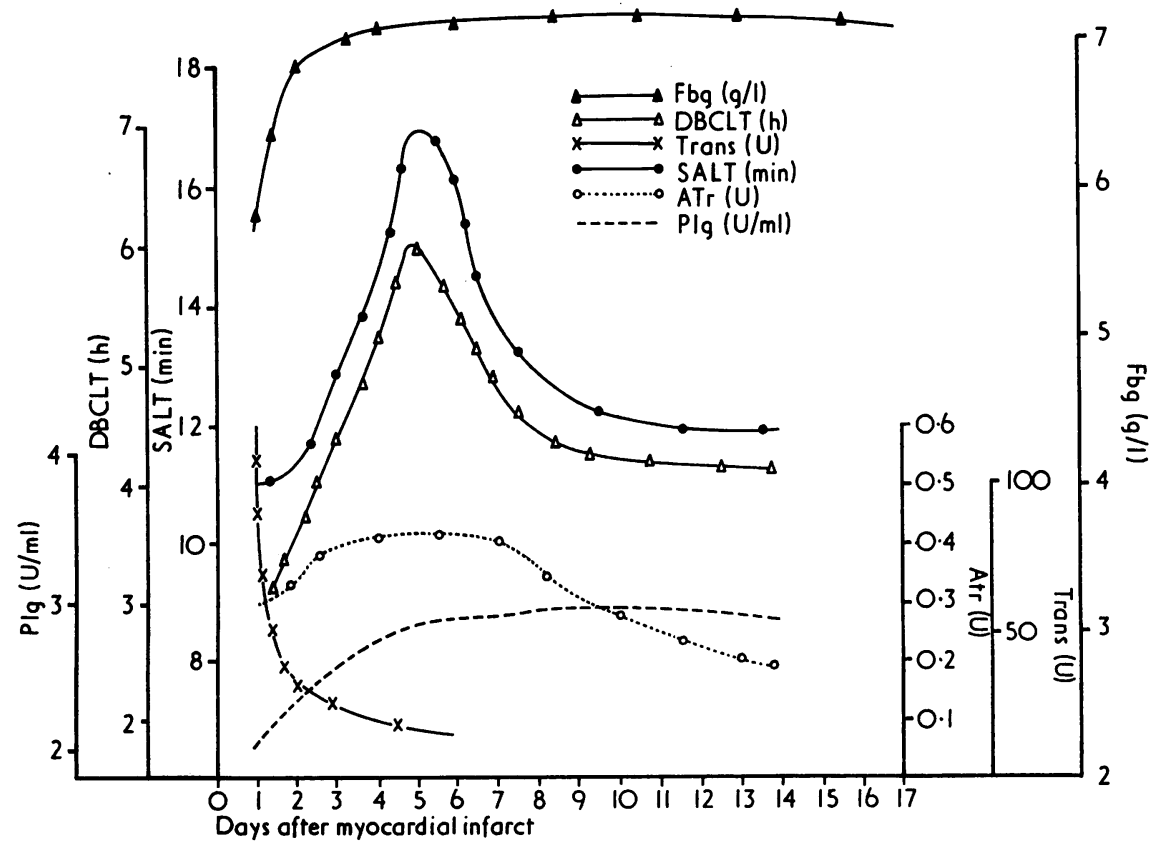

Fig. 6 Changes in the components of the fibrinolytic system in a single typical patient after myocardial infarction. $A T r=$ antitrypsin; $U=$ arbitrary units; Plg = plasminogen; Trans $=S G O T$. 


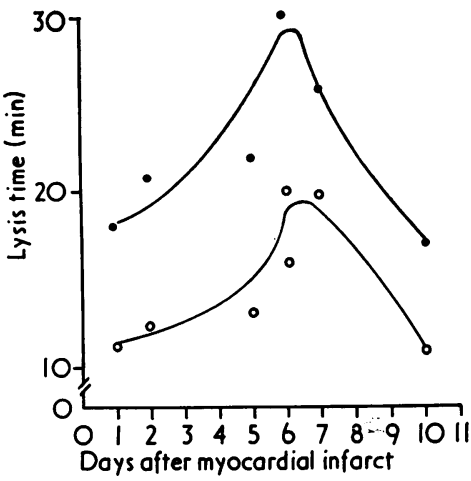

Fig. 7 Comparison of SALT of plasma (O-O-O) with $S A L T$ of plasma with excess of fibrinogen $(16 \mathrm{~g} / \mathrm{l})$

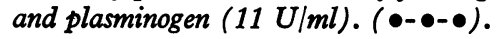

Table Lysis times by the streptokinase-activated lysis time ( $S A L T$ ) and dilute blood clot lysis time (DBCLT) methods in 40 patients at various intervals after myocardial infarction

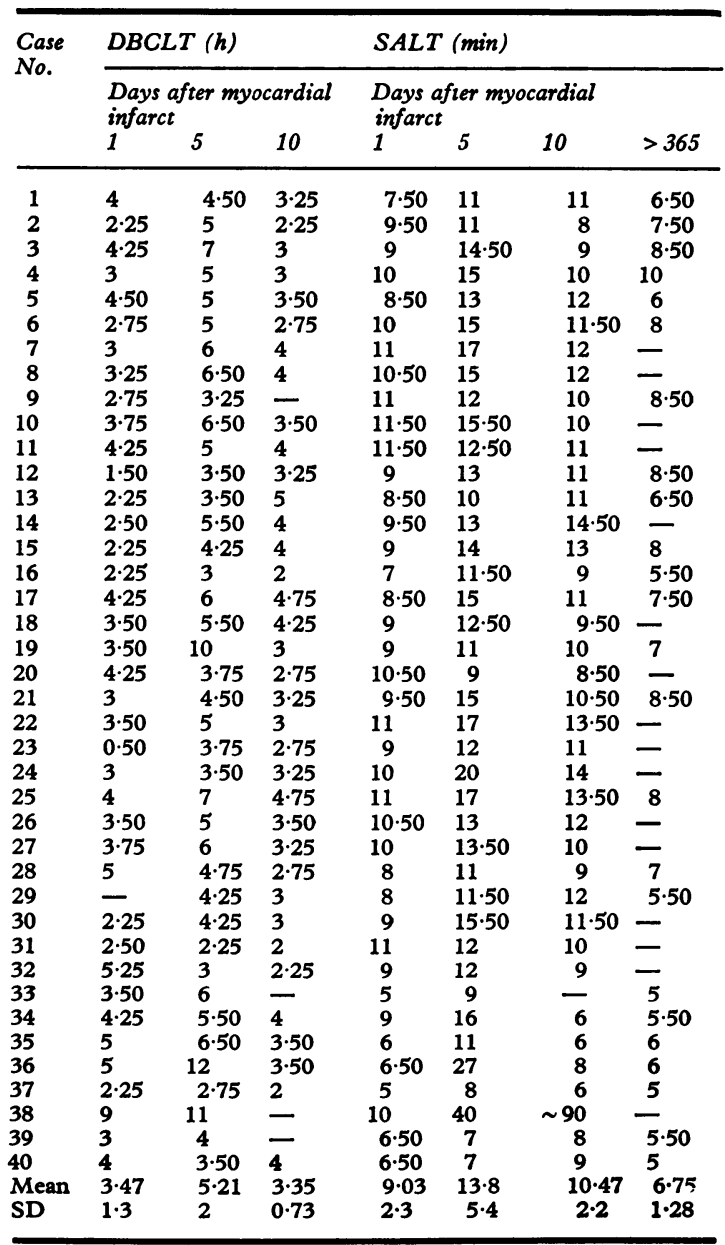

activity (SALT) and fibrinogen level were determined. The mean value for lysis time was $6.75 \pm$ $1.28 \mathrm{~min}$, which was not significantly different from normal values $(P>0.05)$ while the mean fibrinogen level was $2.9 \mathrm{~g} / \mathrm{l}$.

8. The Table summarises the results of both lysis tests on day 1 , on the day of maximum inhibition of fibrinolysis and on day 10 after infarction, as well as the streptokinase activated lysis time in the patients re-examined after 12 to 15 months.

\section{Discussion}

The above results leave little doubt that there is a significant reduction of fibrinolytic activity in the plasma of patients during the first week after an acute myocardial infarction. This reduction, which reaches its maximum 4 to 5 days after the event, is apparently independent of the availability of activators, since it is also evident in the streptokinase activated clot lysis, where an excess of extrinsic activator is supplied. The rise of fibrinogen level is much more prolonged and it remains high after the lysis time approaches normal levels. It may be argued, however, that the higher level of plasminogen on days 5 to 8 counteracts the influence of fibrinogen on lysis with a return of fibrinolytic activity to normal levels. To clarify this point two additional patients were examined by the SALT method, with addition of excess plasminogen and fibrinogen to the plasma. The results (Fig. 7) showed a similar pattern to that obtained in other patients, with a somewhat longer initial lysis time on day 1 and a significant delay on day 5 . This seems to exclude a predominant role of fibrinogen and plasminogen in the observed changes in fibrinolysis on day 5, suggesting that the remaining factors in the fibrinolytic system-the inhibitors-cause this delay.

The level of inhibitors showed a rise and fall which paralleled the delay in lysis time in all the cases in which it was determined, and so appears to be the main factor responsible for the temporarily diminished fibrinolytic activity in subjects after acute myocardial infarction.

One may speculate that there is a transient increase in fibrinolytic activity caused by increased activation immediately after an acute infarction, and that the rise in fibrinogen level as well as that of the inhibitors is a reaction to this increased activity. This assumption will be confirmed or refuted when more sensitive methods for the determination of activators of fibrinolysis become available.

The observation that the fibrinolytic activity was found not to be significantly different from normal 
in the 24 patients re-examined 12 to 15 months after the infarction seems to indicate that the defective fibrinolysis is a transient phenomenon after myocardial infarction rather than a causative factor involved in the pathogenesis of this disease.

\section{References}

Bick, R. L., Bishop, R. C., and Shanbrom, E. S. (1972). Fibrinolytic activity in acute myocardial infarction. American fournal of Clinical Pathology, 57, 359.

Bruhn, H. D., Jipp, P., Okoye, S., and Oltmann, A. (1974). Hypofibrinolyse beim akuten Myokardinfarkt. Medizinische Kli iik, 69, 1951.

Chakrabarti, R., Fearnley, G. R., Hocking, E. D., Delitheos, A., and Clarke, G. M. (1966). Fibrinolytic activity related to age in survivors of myocardial infarction. Lancet, $1,573$.

Chakrabarti, R., Hocking, E. D., Fearnley, G. R., Mann, R. D., Attwell, T. N., and Jackson, D. (1968). Fibrinolytic activity and coronary artery disease. Lancet, 1, 987.

Fearnley, G. R., Balmforth, G., and Fearnley, E. (1957). Evidence of a diurnal fibrinolytic rhythm with a simple method of measuring fibrinolysis. Clinical Science, 16, 645.

Godal, H. C. (1961). Simple syneresis procedure for fibrinogen assay. Scandinavian fournal of Clinical and Laboratory Investigation, 13, 530.

Hume, R. (1958). Fibrinolysis in myocardial infarction. British Heart fournal, 20, 15.
Johnson, A. J., Kline, D. L., and Alkjaersig, M. (1969). Assay methods and standard preparations for plasmin, plasminogen and urokinase in purified systems. Thrombosis et Diathesis Haemorrhagica, 21, 259.

Katz, A. M., McDonald, L., Davies, B., and Edgill, $M$. (1963). Fibrinolysis and blood coagulation in ischaemic heart disease. Lancet, 1, 801.

Merskey, C., Gordon, H., Lackner, H., and Kaplan, B. (1960). Blood coagulation and fibrinolysis in relation to coronary heart disease. British Medical fournal, 1, 219.

Naimi, S., Goldstein, R., and Proger, S. (1963). Studies of coagulation and fibrinolysis of the arterial and venous blood in normal subjects and patients with atherosclerosis. Circulation, 27, 904.

Ogston, D. (1962). Serum mucoproteins and plasma fibrinolytic activity in coronary artery disease. British Medical Fournal, 1, 1242.

Schwert, G. W., and Takenaka, Y. (1955). A spectrophotometric determination of trypsin and chymotrypsin. Biochimica et Biophysica Acta, 16, 570.

Schwick, H. G., Heimburger, N., and Haupt, H. (1966). Antiproteinasen des Humanserums. Zeitschrift für die Gesamte Innere Medizin, 21, 193.

Svehla, C. (1973). Fibrinolytic activity of patients with acute myocardial infarction. American fournal of Clinical Patho$\log y, 59,120$.

Requests for reprints to Dr. E. Gidron, Department of Medicine B, Rothschild University Hospital, Haifa, Israel. 